\title{
Cost of coils for intracranial aneurysms: clinical decision analysis for implementation of a capitation model
}

\author{
Gurpreet S. Gandhoke, MD, MCh, ${ }^{1}$ Yash K. Pandya, ${ }^{2}$ Ashutosh P. Jadhav, MD, ${ }^{3}$ Tudor Jovin, MD, ${ }^{3}$ \\ Robert M. Friedlander, MD, ${ }^{1}$ Kenneth J. Smith, MD, MS, ${ }^{4}$ and Brian T. Jankowitz, MD ${ }^{1}$ \\ Departments of ${ }^{1}$ Neurological Surgery and ${ }^{3}$ Neurology, University of Pittsburgh Medical Center; and ${ }^{4}$ Section of Decision \\ Sciences, Center for Research on Health Care, ${ }^{2}$ School of Medicine, University of Pittsburgh, Pennsylvania
}

\begin{abstract}
OBJECTIVE The price of coils used for intracranial aneurysm embolization has continued to rise despite an increase in competition in the marketplace. Coils on the US market range in list price from $\$ 500$ to $\$ 3000$. The purpose of this study was to investigate potential cost savings with the use of a price capitation model.
\end{abstract}

METHODS The authors built a clinical decision analytical tree and compared their institution's current expenditure on
endovascular coils to the costs if a capped-price model were implemented. They retrospectively reviewed coil and cost
data for 148 patients who underwent coil embolization from January 2015 through September 2016 . Data on the length
and number of coils used in all patients were collected and analyzed. The probabilities of a treated aneurysm being $\leq />$
$10 \mathrm{~mm}$ in maximum dimension, the total number of coils used for a case being $\leq />5$, and the total length of coils used
for a case being $\leq />50 \mathrm{~cm}$ were calculated, as was the mean cost of the currently used coils for all possible combina-
tions of events with these probabilities. Using the same probabilities, the authors calculated the expected value of the
capped-price strategy in comparison with the current one. They also conducted multiple 1 -way sensitivity analyses by
applying plausible ranges to the probabilities and cost variables. The robustness of the results was confirmed by apply-
ing individual distributions to all studied variables and conducting probabilistic sensitivity analysis.

RESULTS Ninety-five $(64 \%)$ of 148 patients presented with a rupture, and $53(36 \%)$ were treated on an elective basis. The mean aneurysm size was $6.7 \mathrm{~mm}$. A total of 1061 coils were used from a total of 4 different providers. Companies A $(72 \%)$ and $B(16 \%)$ accounted for the major share of coil consumption. The mean number of coils per case was 7.3 . The mean cost per case (for all coils) was $\$ 10,434$. The median total length of coils used, for all coils, was $42 \mathrm{~cm}$. The calculated probability of treating an aneurysm less than $10 \mathrm{~mm}$ in maximum dimension was 0.83 , for using 5 coils or fewer per case it was 0.42 , and for coil length of $50 \mathrm{~cm}$ or less it was 0.89 . The expected cost per case with the capped policy was calculated to be $\$ 4000$, a cost savings of $\$ 6564$ in comparison with using the price of Company A. Multiple 1-way sensitivity analyses revealed that the capped policy was cost saving if its cost was less than $\$ 10,500$. In probabilistic sensitivity analyses, the lowest cost difference between current and capped policies was $\$ 2750$.

CONCLUSIONS In comparison with the cost of coils from the authors' current provider, their decision model and probabilistic sensitivity analysis predicted a minimum $\$ 407,000$ to a maximum $\$ 1,799,976$ cost savings in 148 cases by adapting the capped-price policy for coils.

https://thejns.org/doi/abs/10.3171/2017.3.JNS163149

KEY WORDS endovascular; coils; cost savings; capitation model; vascular disorders

$\mathrm{T}$ HE number of intracranial aneurysms treated with endovascular embolization continues to increase both in the US and abroad. Coil embolization still represents the most common form of treatment. Until Micrus was introduced into the market in 2000, Boston Scientific had a monopoly in the detachable coil domain. Coil prices have continued to rise despite an increase in competition in the marketplace. Physicians are in an excellent position to approximate a willingness-to-pay threshold for a unit of new hardware/technology.

The list prices of coils on the US market range from $\$ 500$ to $\$ 3000$. At our center the most commonly used 
TABLE 1. Probabilities and their ranges used in the decision model

\begin{tabular}{lcl}
\hline \multicolumn{1}{c}{ Event } & Probability & Range \\
\hline Aneurysm $\leq 10 \mathrm{~mm}$ & 0.83 & $0.1-0.95$ \\
\hline Aneurysm $\leq 10 \mathrm{~mm} \&$ coils $<5$ & 0.42 & $0.2-1$ \\
\hline $\begin{array}{c}\text { Aneurysm } \leq 10 \mathrm{~mm} \& \text { coils }<5 \& \\
\text { length of coils used }<50 \mathrm{~cm}\end{array}$ & 0.89 & $0.1-1$ \\
\hline $\begin{array}{c}\text { Aneurysm } \leq 10 \mathrm{~mm} \& \text { coils }>5 \& \\
\text { length of coils used }<50 \mathrm{~cm}\end{array}$ & 0.49 & $0.3-0.6$ \\
\hline Aneurysm $>10 \mathrm{~mm} \&$ coils $<5$ & 0.2 & $0.1-0.9$ \\
\hline $\begin{array}{c}\text { Aneurysm }>10 \mathrm{~mm} \& \text { coils }<5 \& \\
\text { length of coils used }<50 \mathrm{~cm}\end{array}$ & 0.33 & $0.25-0.45$ \\
\hline
\end{tabular}

coils are from a company that we shall refer to for purposes of this analysis as Company A. The pricing policy of this company is different from that of another company (referred to as Company B), and this could have major cost implications. We sought to model a cost comparison to show potential cost savings with the use of a price capitation model using Company B products versus Company A products. We used clinical decision analysis to build a decision tree and compared our current expenditure on endovascular coils to the corresponding expenditure if a capped-price model were implemented.

\section{Methods}

We retrospectively reviewed coil and cost data for 148 patients who underwent coil embolization from January 2015 through September 2016. For 128 of the 148 patients, we used coils from our most frequently used provider (Company A). Data on the length and number of coils used in all patients were collected and analyzed. Based on our mean values for treated aneurysm size and total number of coils used and based on the median of the total length of coils used, we calculated the probabilities of a treated aneurysm having a size (maximum dimension) $\leq 10 \mathrm{~mm}$ or $>10 \mathrm{~mm}$ (mean $6.7 \mathrm{~mm}$ ), the total number of coils used for a case being $\leq 5$ or $>5$ (mean 7.3), and the total length of coils used for a case being $\leq 50 \mathrm{~cm}$ or $>50 \mathrm{~cm}$ (median $42 \mathrm{~cm}$ ). These values were selected to best represent the mean/median values from our data. We calculated the mean cost of the currently used coils for all possible combinations of events with the above probabilities.

The total length of coils used was analyzed, because the greater the volume of small-length coils used, the greater the financial advantage to the company. Also, the greater the number of coils used, the longer the procedure and fluoroscopy time. Although we did not take into account any cost other than the hospital cost for the coils, the financial difference associated with coil length is emphasized in our model design.

We used clinical decision analysis techniques to build a decision tree and compared our current expenditure on endovascular coils with that of implementing a capped-price model. In the model, the branch depicting the new cappedprice model used the same events and probabilities as the prevalent strategy to calculate its cost. The cost used under
TABLE 2. Costs and their ranges used in the decision model

\begin{tabular}{|c|c|c|}
\hline Event & Cost (\$) & Range (\$) \\
\hline $\begin{array}{l}\text { Aneurysm } \leq 10 \mathrm{~mm} \& \text { coils } \leq 5 \& \\
\text { length of coils used } \leq 50 \mathrm{~cm}\end{array}$ & 6338 & $3000-6338$ \\
\hline $\begin{array}{l}\text { Aneurysm } \leq 10 \mathrm{~mm} \& \text { coils }>5 \& \\
\text { length of coils used } \leq 50 \mathrm{~cm}\end{array}$ & 11,982 & $5500-11,982$ \\
\hline $\begin{array}{l}\text { Aneurysm }>10 \mathrm{~mm} \& \text { coils } \leq 5 \& \\
\text { length of coils used } \leq 50 \mathrm{~cm}\end{array}$ & 1665 & $800-1665$ \\
\hline $\begin{array}{l}\text { Aneurysm } \leq 10 \mathrm{~mm} \& \text { coils } \leq 5 \& \\
\text { length of coils used }>50 \mathrm{~cm}\end{array}$ & 6761 & $3000-6761$ \\
\hline $\begin{array}{l}\text { Aneurysm } \leq 10 \mathrm{~mm} \& \text { coils }>5 \& \\
\text { length of coils used }>50 \mathrm{~cm}\end{array}$ & 14,263 & $7000-14,263$ \\
\hline $\begin{array}{l}\text { Aneurysm }>10 \mathrm{~mm} \& \text { coils } \leq 5 \& \\
\text { length of coils used }>50 \mathrm{~cm}\end{array}$ & 10,419 & $5000-10,419$ \\
\hline $\begin{array}{l}\text { Aneurysm }>10 \mathrm{~mm} \& \text { coils }>5 \& \\
\text { length of coils used }>50 \mathrm{~cm}\end{array}$ & 12,902 & $6000-12,902$ \\
\hline
\end{tabular}

the capped policy was $\$ 4000$. Company B proposed this price based on their internal data for the mean number of coils used per case on a national basis in relation to their current price per coil. We were not involved in this decision. We report our results on the calculated expected value of the new strategy in comparison with the current one. We also report the results of multiple 1-way sensitivity analyses by applying plausible ranges to the probability and cost variables. We then report the results of a Monte Carlo probabilistic sensitivity analysis, which applies individual distributions to simultaneously examine all variables to confirm the robustness of model results.

Using TreeAge Pro decision-analysis software (TreeAge Software), we constructed a decision model. The following steps depict the components of our decision analysis.

1. The question: Given our calculated probabilities of treating aneurysms with a maximum dimension $\leq 10 \mathrm{~mm}$ or $>10 \mathrm{~mm}$, using a number of coils $\leq 5$ or $>5$ per case, and using a total length of coils $\leq 50 \mathrm{~cm}$ or $>50 \mathrm{~cm}$ for a given case, we sought the cost-minimizing option between using our most commonly used coils from Company A versus switching to the capped-price policy of Company B.

2. The model: We built a decision tree, with the 2 strategies at the square decision node. We added circular chance nodes from each strategy, containing the probabilities of aneurysm size, number of coils used, and total length of coil used until the outcome (the final payoff or total cost of coils used) was reached at the triangular terminal nodes.

3 . The outcome values and probabilities: All probabilities were calculated based on our data from 148 consecutive cases of intracranial aneurysms treated over a period of 21 months. We assigned the hospital cost for coils for each event to the relevant nodes to carry out an economic analysis from the hospital perspective. The costs of coils from Company A reflect true opportunity costs for coils to the hospital and not charges or reimbursement for coils. Table 1 depicts the probabilities and ranges used in the decision model. Table 2 depicts the cost amounts and ranges used in the decision model for the different events.

4. Identify the best strategy: We then "fold back" the decision tree to determine the expected value for each 
TABLE 3. Summary statistics

\begin{tabular}{cc}
\hline Variable & Value \\
\hline Aneurysm size $(\mathrm{mm})$ & \\
\hline Mean & 6.7 \\
\hline SD & 3.5 \\
\hline Range & $2.5-22$ \\
\hline No. of coils/case & 7.3 \\
\hline Mean & 4.9 \\
\hline SD & $1-39$ \\
\hline Range & 10,434 \\
\hline Cost of coils $(\$)$ & 5627 \\
\hline Mean & $1390-26,589$ \\
\hline SD & 42 \\
\hline Range & $5.4-516$ \\
\hline Length of coils $(\mathrm{cm})$ & \\
\hline Median* & \\
\hline Range & \\
\hline
\end{tabular}

* The median is reported for this variable because the SD was more than the calculated mean.

strategy. The favored strategy was the one with the lower cost.

5. One-way sensitivity analysis: We varied each cost and probability individually between best- and worst-case values. We constructed a tornado diagram to depict all 2-way sensitivity analyses in a single image, showing the minimum and maximum differences in costs associated with each parameter's variation.

6. Probabilistic sensitivity analysis: To be able to vary all involved variables simultaneously (in contrast to 1-way sensitivity analysis in which only 1 variable was varied at a time), we performed Monte Carlo probabilistic sensitivity analysis in which 1000 iterations of calculating the cost difference between the 2 strategies were performed. For each of the 1000 iterations, the value for every variable depicted in Tables 1 and 2 was drawn randomly from its constructed distribution. We used beta distributions for probabilities and triangular distributions for cost variables.

7. Assumptions: We assumed that the effectiveness of treating a patient with coils from Company A or Company $\mathrm{B}$ was the same. This was a cost-minimization study. In interpreting the results, we also assumed that all neurointerventionists at our institution would switch to using coils from Company B after implementing the capped policy.

\section{Results}

A total of 148 patients underwent coil embolization for treatment of intracranial aneurysms during the 21-month period from January 2015 through September 2016. These cases included all aneurysms treated with coils during this period. In 95 (64\%) of the 148 cases, the patients presented with a rupture, while 53 patients $(36 \%)$ were treated electively. The mean aneurysm size was $6.7 \mathrm{~mm}$ (SD 3.5 $\mathrm{mm}$, range $2.5-22 \mathrm{~mm}$ ) (Table 3). A total of 1061 coils were used from a total of 4 different providers. Figure 1 graphically displays the number of coils used from each

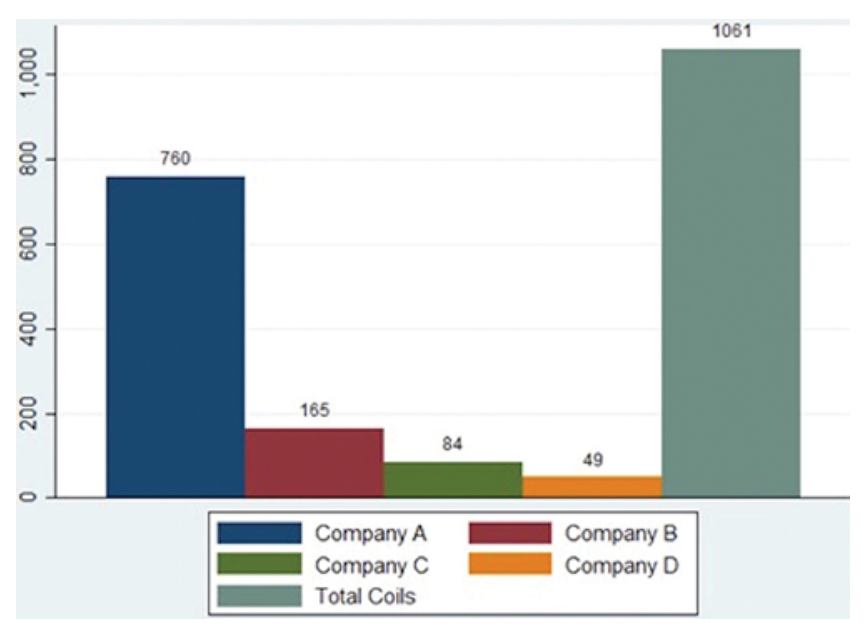

FIG. 1. Bar graph depicting the number of coils used from all providers. Figure is available in color online only.

provider. Companies A (72\%) and B (16\%) accounted for the major share of coil consumption. The mean number of coils used for all cases was 7.3 (SD 4.9, range 1-39). The mean number of coils used per case from Company A only was 5.2 (SD 3.4, range 1-15). The mean cost per case for all coils was \$10,434 (SD \$5627, range \$1390-\$26,589). For the 128 cases in which only coils from Company A were used, the mean cost was $\$ 10,224$ (SD \$5249, range \$1665-\$26,589). Since the standard deviation of the total length of coils used, for all coils, was more than the mean value for that variable, we report the median, which was $42 \mathrm{~cm}$ (range 5.4-516 cm). The median length of Company A coils used was $49 \mathrm{~cm}$ (range 6-516 cm).

The calculated probability of treating an aneurysm $\leq$ $10 \mathrm{~mm}$ was 0.83 , that of using $\leq 5$ coils was 0.42 , and that of using a total coil length $\leq 50 \mathrm{~cm}$ was 0.89 . The expected value of using the capped policy was calculated to be $\$ 4000$, a cost savings of $\$ 6564$ in comparison with using the price of Company A. Figure 2 depicts the decision tree.

\section{Sensitivity Analysis: 1-Way}

Varying all parameters individually through the ranges shown in Tables 1 and 2 yielded just 1 parameter (capitation cost of Company B) that decreased the cost difference between the 2 strategies to zero. The capped policy was cost saving if its cost was less than $\$ 10,500$. With variation within plausible ranges (Tables 1 and 2), none of the other parameters caused the cost of purchasing from Company A to become less than cost with the capped policy. This is depicted as a tornado diagram in Fig. 3.

\section{Sensitivity Analysis: Monte Carlo}

For the multiway analysis, we performed Monte Carlo (probabilistic) sensitivity analysis. Monte Carlo sensitivity analysis can simultaneously vary all uncertain parameters over a range of values and a probability distribution within that range for each parameter, allowing estimation of the relative likelihood of resulting cost differences. In this analysis, parameter values were chosen randomly for 1000 iterations. Beta distributions were constructed for 


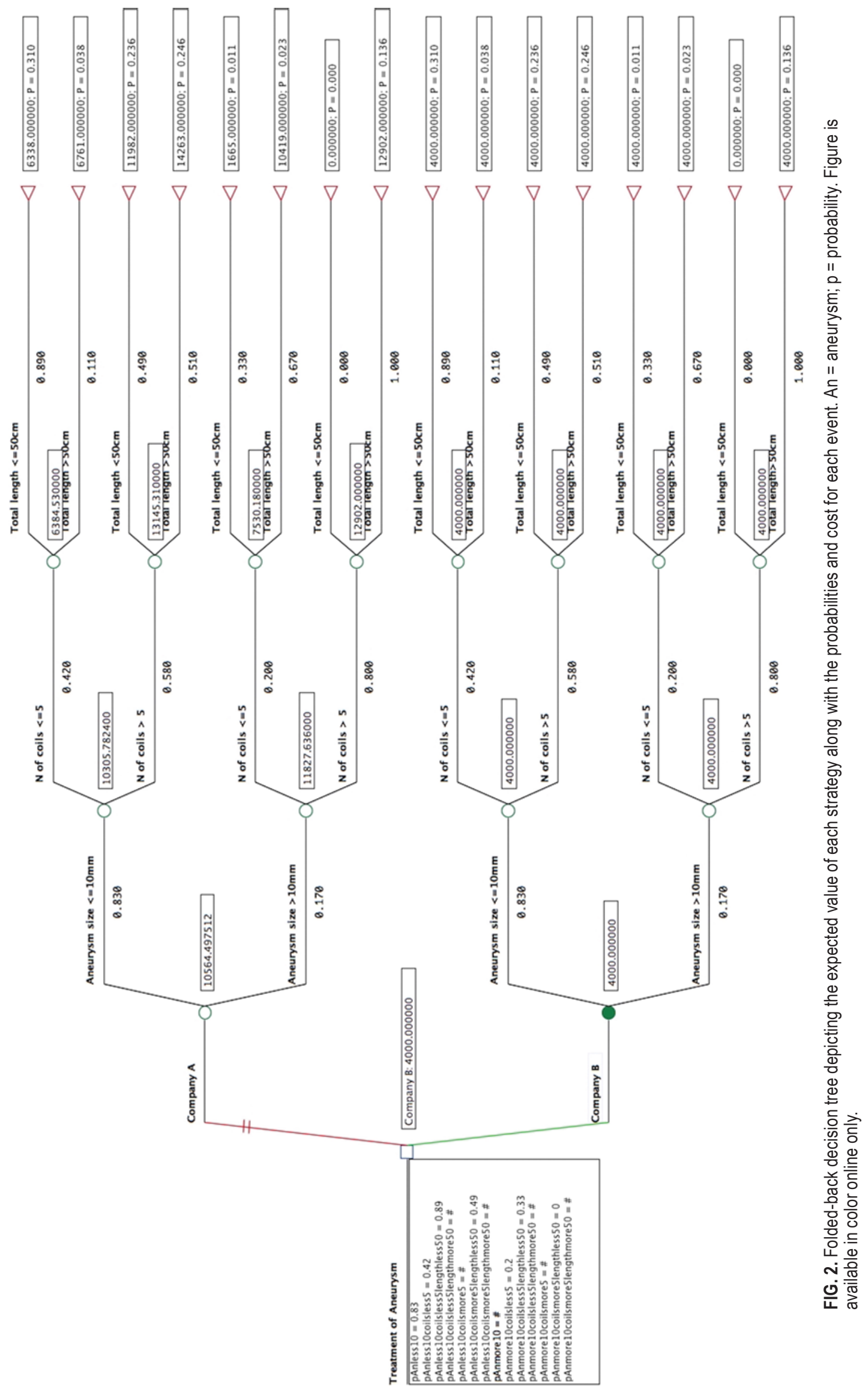


Tornado Analysis (Incremental)

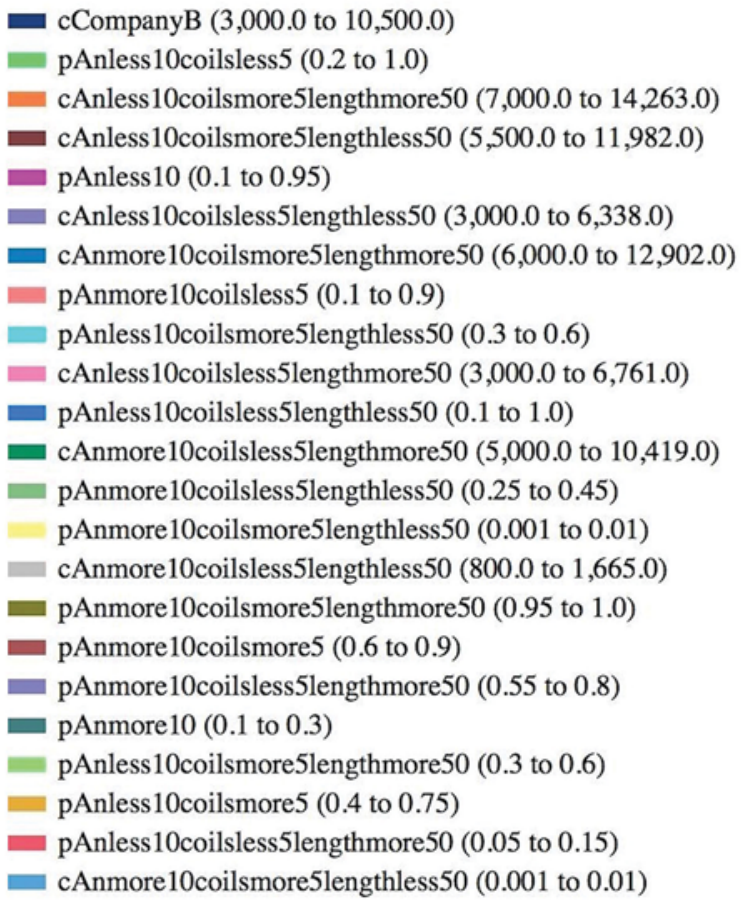

EV: 6564.49751

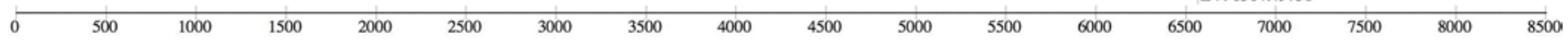

FIG. 3. Tornado diagram depicting multiple 1-way sensitivity analyses of studied variables. An = aneurysm; $c=c o s t ; p=p r o b a b i l-$ ity. Figure is available in color online only.

probabilities, and for costs we used triangular distributions $($ commonest $=$ baseline value, minimum and maximum ends of the ranges in our cost data). In this analysis, the lowest cost difference seen was $\$ 2750$. The cumulative probability of these cost differences is graphed in Fig. 4.

Monte Carlo probabilistic sensitivity analysis thus predicted a total cost savings in 148 cases ranging from a minimum of $\$ 407,000$ to a maximum of $\$ 1,799,976$.

\section{Discussion}

In our current health system, we are increasingly required to prove value in what we do. Frequently, more is spent during a hospitalization for an uncomplicated endovascular treatment of an unruptured aneurysm than its Medicare reimbursement. All costs in excess of this reimbursement represent a financial loss to the institution. The more expensive the coil, the more rapidly this loss multiplies.

A 2012 article in the New England Journal of Medicine argues that when physicians consider costs, they are indeed serving the real interests of individual patients. ${ }^{5}$ It is now abundantly evident that physicians and hospitals are dependent on each other's financial viability. Supplies and devices accounted for $24 \%$ of the increase in hospital costs 


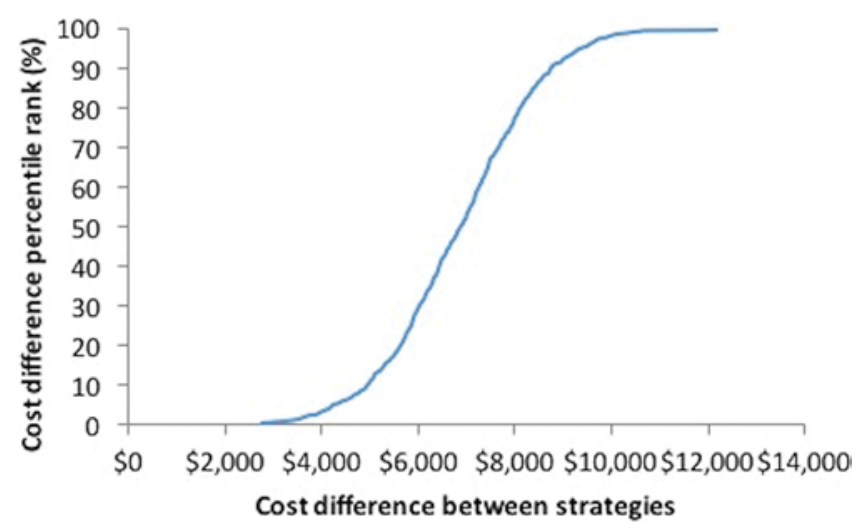

FIG. 4. Cumulative likelihood of cost difference between buying from Company $A$ and using the capped policy. Figure is available in color online only.

per discharge from 2001 to $2006,{ }^{2}$ so it is important that hospitals begin to partner with physicians to target supply and device expenses.

Governments, policy makers, researchers, and payers are now relying on cost studies to make decisions on the value of new modalities. We performed a cost-minimization study and calculated the economic impact of implementing a capped-price policy versus paying per coil at our institution. We used the hospital perspective and based our calculations on the cost of coils to the hospital. Costs reflect true opportunity costs to the hospital for coils bought and not charges or reimbursement for coil cost. We assumed that the effectiveness of using coils from either strategy was the same.

Clinical decision models and Monte Carlo probabilistic analysis are very strong tools for comparing the costs of 2 strategies with similar effectiveness. Since we used both 1-way and multiway probabilistic sensitivity analyses, wherein all involved variables were ranged with an exhaustive set of plausible ranges, the values of an aneurysm size of $\leq>>10 \mathrm{~mm}$, total number of coils used of $\leq>>5$, and total length of coils used of $\leq>>50 \mathrm{~cm}$ served as good markers to calculate our initial probabilities. These values were also not selected arbitrarily but were based on the means (aneurysm size and total coils used) or median (length of coil used) from our own data set. Since $7 \mathrm{~mm}$ is the average size of a treated aneurysm, one could argue that the base case aneurysm size should be $7 \mathrm{~mm}$ rather than $10 \mathrm{~mm}$. The advantage of using the Monte Carlo probabilistic sensitivity analyses is that it accounts for the probabilities of an aneurysm being less than $10 \mathrm{~mm}$, with the probabilities being drawn from a distribution of aneurysm size. This exhaustively accounts for the situation in which an aneurysm is $\leq />7 \mathrm{~mm}$ and $\leq />10 \mathrm{~mm}$.

Kashlan et al. ${ }^{1}$ reported on an estimated cost savings of $\$ 76,732$ after implementing a standardized coil price, calculated by dividing the total cost of all coils used in each 6-month period by the number of coils used. As an example, they state, "Coil A, which may cost \$200 from Vendor 1, $\$ 400$ from Vendor 2, $\$ 1000$ from Vendor 3, and $\$ 800$ from Vendor 4 , would have an average shelf price of $\$ 600$." It is interesting to note that all these vendors agreed to the policy (without agreeing to publish the specifics).
This gives an idea of the cost savings that might be possible if we as physicians are willing to negotiate with the companies to bring down the cost of their products. In our experience, Company B readily agreed to the implementation of the capped policy. Also of interest was Kashlan and colleagues' finding of an almost immediate maximal effect in cost savings achieved by logging and reporting opened but unused items. We found the mean number of opened but not used coils over 148 cases was 0.7 , a number small enough for us not to include it in the analytical model. We, as a rule, have always logged and reported all opened but unused items.

Cost-reduction plans such as implementing a capitation model often raise the concern that enforced pricing will limit the type of coils available to the neurointerventionists. The method of detachment, perception of efficacy, softness, etc., do influence coil choice, but no study to date has shown any single coil to be more efficacious than any other. The existing randomized trials of bioactive coils have also failed to demonstrate any difference in outcome. A long-standing concern is what to do with companies that refuse to participate, especially when their products are preferred or perceived as superior. Trials, including the MAPS (Matrix and Platinum Science) trial ${ }^{3}$ and the Cerecyte trial, ${ }^{4}$ have failed to show differences in efficacy or recurrence based on coil type. The HELPS (hydrogelcoated coils vs bare platinum coils) trial ${ }^{6}$ demonstrated that hydrogel-coated coils did not reduce late aneurysm rupture or improve outcome compared with bare-metal coils. We are moving toward a total implementation of a capped policy, which will add to the competition and incentivize other companies to follow the example of Company B. Thus, all coils should become available to the surgeon at a more economical cost.

It is easy to perceive that Company B appears to have an incentive to offer more favorable pricing, but it is reasonable to assume that prices are easily obtained between institutions and that when a price is marketed to an institution, however discretely, that price will become common knowledge and used by other institutions as leverage. In our opinion, it behooves all companies to offer the lowest price possible to attain new market share or an increase in their current share.

We did not vary the coil costs of Company A above the calculated mean for each studied event. We did, however, vary the cost below the calculated mean. Despite this conservative comparison, for all iterations and in all possible sensitivity analyses, it emerged as the not-preferred policy.

No matter what the probability of treating an aneurysm $\leq />10 \mathrm{~mm}$, using a number of coils $\leq />5$, or using a total length of coils $\leq />50 \mathrm{~cm}$, it was more economical to use the capped amount of $\$ 4000$ in comparison with the coils from Company A, and this remained the dominant strategy up to a cost of $\$ 10,500$. This substantiates the significant cost difference between the 2 strategies and explains the resulting cost savings.

The following limitations of our study must be taken into consideration when interpreting the results. We chose not to discuss rates of occlusion, complications, or recanalization and the attending cost, as we presumed equal efficacy between the coils. We do plan to explore complica- 
tions and recanalization in a future manuscript. Currently we do not have enough follow-up. There was a lack of blinding to determine the coil to be used by the surgeon.

We treated all aneurysms to complete occlusion to the best of our abilities. No aneurysm was intentionally subtotally coiled. We have not retreated a single patient from this cohort, and we continue to follow the patients both angiographically and clinically.

The findings from this study serve as an impetus to explore further cost-saving strategies in aneurysm coiling. A few strategies that we are exploring include use of different catheters and wires, observation of patients with unruptured aneurysms in a stepdown unit instead of an intensive care unit, judicious use of follow-up imaging, and treating large aneurysms with flow diverters instead of coiling. Clinical decision analysis also opens doors to explore other opportunities to save costs in endovascular therapy, such as in carotid artery stenting (comparison of stents, balloons, wires, distal protection devices) and thrombectomy (use of stent retrievers vs aspiration).

\section{Conclusions}

In a comparison of a capped-price policy versus the cost of coils from our current provider, our decision model and probabilistic sensitivity analysis predicted a minimum of $\$ 407,000$ to a maximum of $\$ 1,799,976$ in cost savings in 148 patients through adoption of a In a comparison of a capped-price policy for coils. This study should provide impetus to explore coil cost capitation as a cost-minimization strategy in endovascular surgery for coil embolization of intracranial aneurysms.

\section{References}

1. Kashlan ON, Wilson TJ, Chaudhary N, Gemmete JJ, Stetler WR Jr, Dunnick NR, et al: Reducing costs while maintaining quality in endovascular neurosurgical procedures. J Neurosurg 121:1071-1076, 2014

2. Maeda JL, Raetzman SO, Friedman BS: What hospital inpatient services contributed the most to the 2001-2006 growth in the cost per case? Health Serv Res 47:1814-1835, 2012
3. McDougall CG, Johnston SC, Gholkar A, Barnwell SL, Vazquez Suarez JC, Massó Romero J, et al: Bioactive versus bare platinum coils in the treatment of intracranial aneurysms: the MAPS (Matrix and Platinum Science) trial. AJNR Am J Neuroradiol 35:935-942, 2014

4. Molyneux AJ, Clarke A, Sneade M, Mehta Z, Coley S, Roy $\mathrm{D}$, et al: Cerecyte coil trial: angiographic outcomes of a prospective randomized trial comparing endovascular coiling of cerebral aneurysms with either cerecyte or bare platinum coils. Stroke 43:2544-2550, 2012

5. Rosenbaum L, Lamas D: Cents and sensitivity-Teaching physicians to think about costs. N Engl J Med 367:99-101, 2012

6. White PM, Lewis SC, Gholkar A, Sellar RJ, Nahser H, Cognard C, et al: Hydrogel-coated coils versus bare platinum coils for the endovascular treatment of intracranial aneurysms (HELPS): a randomised controlled trial. Lancet 377:1655-1662, 2011

\section{Disclosures}

Dr. Jovin reports a consultant relationship with Anaconda and Johnson \& Johnson and direct stock ownership in Silk Road Medical and Blockade Medical.

\section{Author Contributions}

Conception and design: Jankowitz, Jadhav, Gandhoke. Acquisition of data: Jankowitz, Gandhoke, Pandya. Analysis and interpretation of data: Gandhoke, Jadhav, Smith. Drafting the article: Gandhoke, Friedlander. Critically revising the article: all authors. Reviewed submitted version of manuscript: Jankowitz, Gandhoke, Jadhav, Jovin, Friedlander, Smith. Approved the final version of the manuscript on behalf of all authors: Jankowitz. Statistical analysis: Gandhoke, Smith. Administrative/technical/material support: Jankowitz, Gandhoke, Jovin, Friedlander. Study supervision: Jankowitz, Jadhav, Jovin, Friedlander, Smith.

\section{Correspondence}

Brian T. Jankowitz, Department of Neurological Surgery, University of Pittsburgh Medical Center, 200 Lothrop St., Ste. B400, Pittsburgh,PA 15213. email: jankbt@upmc.edu. 